Polibotánica

ISSN electrónico: 2395-9525

POLIBETÁNICA polibotanica@gmail.com Instituto Politécnico Nacional México http:www.polibotanica.mx

\title{
ÁCIDO ROSMARÍNICO, FENÓLICOS TOTALES Y CAPACIDAD ANTIOXIDANTE EN TRES VARIEDADES DE Ocimum basilicum L. CON DIFERENTES DOSIS DE POTASIO
}

\section{ROSMARINIC ACID, TOTAL PHENOLICS AND ANTIOXIDANT CAPACITY IN THREE VARIETIES OF Ocimum basilicum L. WITH DIFFERENT POTASSIUM DOSES}

Ramírez-Aragón, M.G.; V.J. Borroel-García, L. Salas-Pérez, J.D. López-Martínez, M.A. Gallegos-Robles, y H.I. Trejo-Escareño.

ÁCIDO ROSMARÍNICO, FENÓLICOS TOTALES Y CAPACIDAD ANTIOXIDANTE EN TRES VARIEDADES DE Ocimum basilicum L. CON DIFERENTES DOSIS DE POTASIO. ROSMARINIC ACID, TOTAL PHENOLICS AND ANTIOXIDANT CAPACITY IN THREE VARIETIES OF Ocimum basilicum L. WITH DIFFERENT POTASSIUM DOSES.

POLIBETÁNICA Instituto Politécnico Nacional
Núm. 47: 8[-98 México. Enero 2019

DOI: $10.18387 /$ polibotanica.47.7 


\section{ÁCIDO ROSMARÍNICO, FENÓLICOS TOTALES Y CAPACIDAD ANTIOXIDANTE EN TRES VARIEDADES DE Ocimum basilicum L. CON DIFERENTES DOSIS DE POTASIO.}

\section{ROSMARINIC ACID, TOTAL PHENOLICS AND ANTIOXIDANT CAPACITY IN THREE VARIETIES OF Ocimum basilicum L. WITH DIFFERENT POTASSIUM DOSES.}

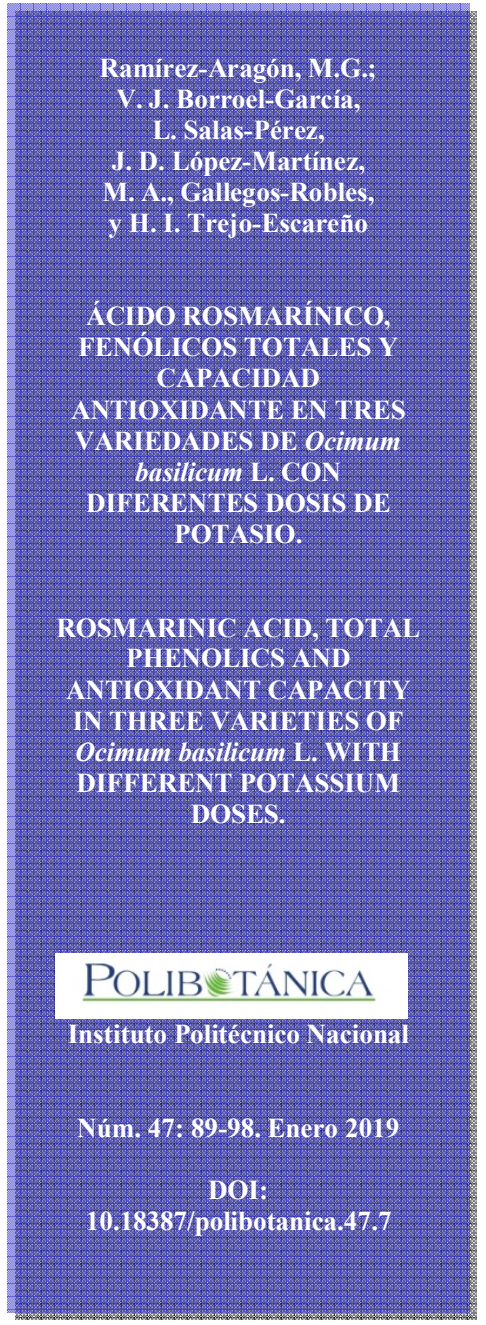

M. G. Ramírez-Aragón

Facultad de Agricultura y Zootecnia. Universidad Juárez del Estado de Durango. Carretera Gómez Palacio-Tlahualilo Km. 32. Venecia, Gómez Palacio, Dgo., México.

\section{J. Borroel-García / vjbg1979@gmail.com}

L. Salas-Pérez

Universidad Politécnica de Gómez Palacio. Carretera La Torreña Km 820, Localidad El Vergel CP 35120, Gómez Palacio, Dgo., México

J. D. López-Martínez

M. A., Gallegos-Robles

H. I. Trejo-Escareño

Facultad de Agricultura y Zootecnia. Universidad Juárez del Estado de Durango. Carretera Gómez Palacio-Tlahualilo Km. 32. Venecia, Gómez Palacio, Dgo., México.

RESUMEN: El objetivo de esta investigación fue determinar el contenido de ácido rosmarínico (AR), compuestos fenólicos totales (CFT) y capacidad antioxidante equivalente en Trolox (CAET) en tres variedades de albahaca (Ocimum basilicum L.) aplicando dosis diferentes de potasio en solución nutritiva $\left(7,9,11\right.$ y $\left.13 \mathrm{mmol} \mathrm{L}^{-1}\right)$. Se utilizó un diseño factorial $3 \times 4$ en bloques al azar, donde los factores fueron genotipo y concentración de potasio. Todas las variables analizadas presentaron diferencia significativa $(p \leq 0.01)$. El contenido de AR estuvo presente en un rango de 0.4 a 6.4

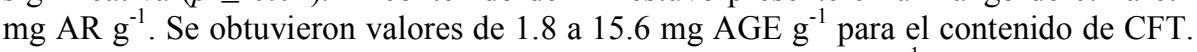
Los resultados obtenidos de CAET fueron de 39 a $79.9 \mathrm{mg} \mathrm{g}^{-1}$ y estos valores se incrementaron en relación al contenido de ácido rosmarínico y compuestos fenólicos totales. La variedad con mayor contenido de ácido rosmarínico, fenólicos totales y capacidad antioxidante fue Red Rubin a $13 \mathrm{mmol} \mathrm{L}^{-1}$. El manejo de dosis de $\mathrm{K}$ en diferentes variedades de albahaca favorece la síntesis de metabolitos secundarios importantes para diferentes áreas de ciencia, por lo que el incremento de la producción de albahaca y de su calidad nutracéutica podría ser una alternativa para abrir nuevos mercados enfocados a la industria alimentaria y medicinal.

Palabras clave: albahaca, metabolitos secundarios, tratamientos, variedades.

ABSTRACT: The objective of this study was to determine the content of rosmarinic acid (RA), total phenolic compounds (TPC) and antioxidant capacity in Trolox (ACET) in three varieties of basil (Ocimum basilicum L.) employing different doses of potassium in nutrient solution $\left(7,9,11\right.$ and $\left.13 \mathrm{mmol} \mathrm{L}^{-1}\right)$. It was used a $3 \times 4$ factorial random block design, where the factors were genotype and concentration of potassium. All the variables analyzed presented a significant difference $(\mathrm{p} \leq 0.01)$. The AR content presented a range of 0.4 to $6.4 \mathrm{mg} \mathrm{AR} \mathrm{g}^{-1}$. Values of 1.8 to $15.6 \mathrm{mg} \mathrm{AGE} \mathrm{g}^{-1}$ were obtained for TPC content. The results obtained from ACET were 39 to $79.9 \mathrm{mg} \mathrm{g}^{-1}$ and these values were increased in relation to rosmarinic acid content and total phenolic compounds. The variety with the highest content of rosmarinic acid, total phenolics and 
antioxidant capacity was Red Rubin at $13 \mathrm{mmol} \mathrm{L}^{-1}$. The management of $\mathrm{K}$ doses in different varieties of basil supports the synthesis of secondary metabolites that are important for different areas of science, on this wise the increase of basil production and its nutraceutical quality would be an alternative to open new markets focused on food and medical industry.

Key words: basil, secondary metabolites, treatments, varieties.

\section{INTRODUCCIÓN}

La albahaca (Ocimum basilicum L.) es una especie medicinal y aromática originaria de la India, África y Asia meridional que actualmente se cultiva en muchos países del mundo (NurzynskaWierdak, 2013). Esta planta se utiliza no sólo para cocinar, sino también en la elaboración de fragancias comerciales, saborizantes y para aumentar la vida útil de algunos alimentos (LópezBlancas et al., 2014). La albahaca es ampliamente utilizada en la medicina tradicional como tónico digestivo y para curar inflamaciones y resfriados Los extractos de albahaca tienen propiedades anticonvulsionantes, antimicrobiana y antifúngica (Nguyen et al., 2010; Parr \& Bolwell, 2000). Estas propiedades son atribuidas a los altos niveles de compuestos fenólicos entre ellos los ácidos fenólicos como el ácido rosmarínico, el cual contribuye a la capacidad antioxidante de la planta (Kwee \& Niemeyer, 2011; Lee \& Scagel, 2009; Nguyen \& Niemeyer, 2008).

En México, la producción de albahaca es de 4,589 ton, en una superficie de 476.6 hectáreas (SIAP, 2015). Los estados productores de albahaca son Baja California, Baja California Sur, Morelos, Nayarit, Puebla y Quintana Roo, de los cuales el primero utiliza sistemas controlados de producción en invernaderos donde existen parámetros establecidos óptimos para el desarrollo del cultivo tales como humedad, temperatura y agua del suelo y el resto sistemas convencionales a campo abierto. Estas entidades cultivan 38.5, 281, 48.1, 48, 60 y 1 hectáreas, respectivamente. Baja California Sur, el estado con mayor nivel de producción, destina su cosecha a la exportación (SIAP, 2015). El potencial de rendimiento de albahaca en otros estados del país es amplio; por lo que, abrir espacios para incrementar este cultivo cuidando y controlando el desarrollo de la planta; obteniendo con ello, un aumento en su calidad nutracéutica desde el punto de vista de capacidad antioxidante ya que podría ser una alternativa para mercados orientados a la industria alimentaria y medicinal (Sanchez \& Lucero, 2012). Sin embargo, dada la variabilidad genética y las condiciones de crecimiento que puede tener esta planta en la composición bioquímica cuando es cosechada, se deben realizar estudios considerando la variedad a utilizar y las condiciones de crecimiento para determinar cual genotipo es el más indicado, para la extracción de compuestos con actividad biológica (José et al., 2014). Uno de los factores más importantes para el crecimiento de las plantas es la disponibilidad de macronutrientes ya que no solo afecta la formación de biomasa y el subsecuente rendimiento, sino también la producción de compuestos del metabolismo secundario como los compuestos fenólicos (Nguyen et al., 2010), estos compuestos son de gran interés ya que cuando son incluidos en la alimentación presentan actividad biológica, debido a que intervienen en el equilibrio celular, considerándose antioxidantes (Treutter, 2006). Por ello es necesario su estudio para hacer una relación entre el cuidado del cultivo y el desarrollo de los metabolitos secundarios que se producen en la planta. Debido a esto, existen estudios que han señalado que el control de los nutrientes como el potasio $(\mathrm{K})$ durante el crecimiento de las plantas tiene un efecto significativo en la producción de compuestos fenólicos, particularmente el ácido rosmarínico (Nguyen \& Niemeyer, 2008). El K es un nutriente esencial que contribuye en el crecimiento, rendimiento y en el contenido de aceite esencial cuando se combina con otros macronutrientes como nitrógeno, fósforo, magnesio y calcio (Singh et al., 2004). Nguyen et al. (2010), demostraron que los cambios en la dosis de potasio pueden afectar significativamente la composición fenólica y la capacidad antioxidante de hojas de albahaca. La tasa más alta de potasio utilizada para su estudio fue de $5.0 \mathrm{mM}$ de $\mathrm{K}$, esta concentración produjo valores más altos de ácido chicórico y rosmárinico. Por lo tanto, el objetivo de este estudio fue aportar información nueva en el contenido de ácido rosmarínico, compuestos fenólicos totales y 
capacidad antioxidante total en tres variedades de albahaca (Ocimum basilicum L.) aplicando diferentes dosis diferentes de potasio en la solución nutritiva.

\section{MÉTODOS}

\section{Lugar de establecimiento del experimento}

El trabajo se llevó a cabo en un invernadero semiautomático ubicado en la Universidad Politécnica de Gómez Palacio, Durango, (25³8'19.83'’N -103³1'52.12'’O). Los valores de temperatura y humedad relativa del invernadero se mantuvieron entre 25 a $30{ }^{\circ} \mathrm{C}$ y $70-80 \%$, respectivamente.

\section{Materiales vegetales y condiciones de crecimiento}

Se utilizaron las variedades de albahaca Sweet Lemon, Cinammon y Red Rubin. La siembra se realizó en bandejas de germinación de poliestireno de 250 cavidades y se utilizó peat moss como sustrato. Las bandejas se regaron dos veces al día con agua hasta el día del trasplante. Cuando las plántulas presentaron tres a cuatro hojas verdaderas y una altura de $10 \mathrm{a} 15 \mathrm{~cm}$ se trasplantaron en macetas de plástico negro de un galón de capacidad, las cuales contenían una mezcla de arena: perlita (80:20). Se utilizó un diseño factorial $(3 \times 4)$ en bloques al azar, donde los factores fueron: genotipo y concentración de potasio. Se realizaron tres repeticiones por tratamiento.

\section{Preparación de soluciones nutritivas}

Para la preparación de las soluciones nutritivas se utilizó el método universal de Steiner (Steiner, 1961). Respecto al K (KOH reactivo marca Jalmek, Mexico) se tomó como control la concentración de $7 \mathrm{mmol} \mathrm{L}^{-1}$ y se hicieron los ajustes para incrementar la cantidad de $\mathrm{K}$ en las diferentes concentraciones a aplicar en el experimento $\left(7,9,11\right.$ y $\left.13 \mathrm{mmol} \mathrm{L}^{-1}\right)$. Los fertilizantes utilizados fueron $\mathrm{MgO}, \mathrm{CaSO}_{4}, \mathrm{H}_{3} \mathrm{PO}_{4}$, UREA, $\mathrm{KOH}$ (reactivos marca Jalmek esta ultima sal utilizada como fuente de $\mathrm{K}$. Así mismo, se tomó en cuenta la composición química del agua de riego, para determinar la contribución de aniones y cationes por análisis fisicoquímico de acuerdo a la normativa mexicana, la cantidad de iones de potasio en el agua fue de $0.01 \mathrm{mmol}-\mathrm{L}^{-1}$.

\section{Aplicación de las soluciones nutritivas y cosecha}

La aplicación regular de la solución nutritiva comenzó en la fecha del trasplante y se realizó dos veces al día regando cada maceta con $0.250 \mathrm{~L}$. La cosecha se realizó 50 días después de trasplante.

\section{Preparación de las muestras y obtención de extractos}

Las plantas de albahaca fresca fueron lavadas con agua potable y después se depositaron sobre papel canela y se dejaron secar a temperatura ambiente $\left(25 \pm 2^{\circ} \mathrm{C}\right)$ durante 15 días. Las muestras fueron molidas en licuadora (Hamilton Beach) y almacenadas a $5^{\circ} \mathrm{C}$ para la posterior obtención de extractos (Ramos De León, Sanabria Chópite, Rodríguez González, \& Ulacio, 2012). Se realizó una extracción sólido-líquido mediante la adición de $0.5 \mathrm{~g}$ de la muestra en 5 $\mathrm{ml}$ de etanol. Se utilizó un agitador "Stuart" para mantener la mezcla bajo agitación durante 24 horas a temperatura ambiente $\left(25 \pm 2^{\circ} \mathrm{C}\right)$. Después de 24 horas, el vacío de la muestra se filtró y se evaporó el etanol (marca ITEMS 96\%, México) con rotavapor (Buchí, Modelo-210) y un baño de agua (RIOSA, México) a $35-40^{\circ} \mathrm{C}$. Los extractos obtenidos se almacenaron en ultracongelación a $-20^{\circ} \mathrm{C}$ hasta su análisis.

\section{Determinación de ácido rosmarínico}

La separación y cuantificación de ácido rosmarínico se realizó por cromatografía líquida de alta resolución (HPLC, Agilent Technologies, USA.) usando condiciones inicialmente desarrollados por Shan et al. (2005). El método fue descrito en detalle previamente por Nguyen y Niemeyer (2008). Este método se adaptó a las condiciones con las que cuenta el equipo del laboratorio, en 
el cual se usó un gradiente de dilución con ácido fórmico (Sigma Aldrich) al 10\% (dilución A) y $80 \%$ de metanol (ITEMS, México) (dilución B) fases móviles. El HPLC está equipado con un flujo de inyección de $5 \mu \mathrm{L}$ y una columna C-18 marca Agilent Technologies, USA. (5 lm, 4,6 $\mathrm{mm}$ x $50 \mathrm{~mm}$ ). Antes del análisis, los extractos reconstituidos con etanol al $96 \%$ en escala 1:10 $\mathrm{ml}$ de albahaca se filtraron utilizando microdiscos (Millex GS) de 45 micras de poro. Se identificó el ácido rosmarínico (AR) utilizando absorbancia con una longitud de onda a $280 \mathrm{~nm}$ por comparación de los tiempos de retención de cromatografía contra estándares analíticos de la marca Sigma Aldrich, el AR se cuantificó por comparación de áreas de los picos integrados para las curvas de calibración externas y se reportó como miligramo de ácido rosmarínico por gramo de muestra (mg AR g $\left.{ }^{-1}\right)$.

\section{Determinación de compuestos fenólicos totales}

El contenido de compuestos fenólicos totales (CFT) se midió usando una modificación del método de Folin-Ciocalteu (Ainsworth \& Gillespie, 2007). $50 \mu \mathrm{L}$ de extracto reconstituido se mezclaron con $3 \mathrm{ml}$ de agua destilada en un tubo de ensayo. Se agregaron $250 \mu \mathrm{L}$ de reactivo de Folin-Ciocalteu (Sigma-Aldrich, St. Louis MO, EE.UU.), se agitó en vórtex durante $10 \mathrm{~s}$. Después de 3 minutos de reacción se añadieron $750 \mu \mathrm{L}$ de carbonato de sodio $(20 \% \mathrm{p} / \mathrm{v})$ y se agitaron durante $10 \mathrm{~s}$, seguido se añadieron $950 \mu \mathrm{L}$ de agua destilada, se agitó nuevamente en vortex y se dejó reaccionar durante 2 horas a temperatura ambiente y en un lugar oscuro. Para la obtención de absorbancia de la solución se leyó a $765 \mathrm{~nm}$ en un espectrofotómetro Genesys (USA) 10 UV. El contenido fenólico se calculó utilizando una curva de calibración usando ácido gálico como estándar, y los resultados fueron reportados en $\mathrm{mg}$ de ácido gálico equivalente por gramo de muestra $\left(\mathrm{mg} \mathrm{AGE} \mathrm{g}^{-1}\right)$. Los análisis se realizaron por triplicado.

\section{Determinación de capacidad antioxidante total}

El ensayo de $\mathrm{ABTS}^{+}$se realizó de acuerdo a la metodología propuesta por Aubad et al. (2007). El radical se genera por una reacción de oxidación del ABTS (Sigma aldrich, USA.) con persulfato de potasio. En la evaluación se utilizan $20 \mu \mathrm{L}$ de muestra y $980 \mu \mathrm{l}$ de la solución de $\mathrm{ABTS}^{+}$en buffer fosfato a un $\mathrm{pH}$ de 7.4. Luego de 30 minutos se lee la absorbancia (734 nm) por espectrofotometría. La curva de calibración se realizó usando Trolox como estándar y se expresaran los resultados como capacidad antioxidante total en equivalentes Trolox miligramo por gramo de muestra (CAET $\mathrm{mg} \mathrm{g}^{-1}$ ).

\section{Análisis estadístico}

El análisis estadístico se llevó a cabo mediante un análisis de varianza utilizando el programa estadístico SAS (2004) y la comparación de medias se realizó usando la prueba de Tukey con un valor de significancia de 0.05 .

\section{RESUlTADOS}

\section{Contenido de ácido rosmarínico}

El contenido de ácido rosmarínico (AR) obtenido en el presente estudio mostró diferencias significativas para los factores estudiados $(p \leq 0.01)$ (tabla 1). Para el factor genotipo, Red Rubin (RR) presentó el valor más alto de AR, siendo 62\% mayor respecto a la variedad con menor concentración, seguido por la variedad Cinammon y por ultimo Sweet Lemon. Para el factor dosis de potasio, puede observarse que la concentración de AR aumenta con el incremento del $\mathrm{K}$ en un $93 \%$, comparando el control con la dosis más alta. 
Tabla 1. Valores promedios por variedad y dosis de potasio de ácido rosmarínico, compuestos fenólicos totales y capacidad antioxidante total.

\begin{tabular}{lccc}
\hline & AR $\left(\mathbf{m g ~ g}^{-1}\right)$ & CFT $\left(\mathbf{m g ~ A G E ~} \mathbf{~}^{-1}\right)$ & CAET $_{\left(\mathbf{m g ~ g}^{-1}\right)}$ \\
\hline Variedad & & & \\
SL & $1.536 \mathrm{c}$ & $7.395 \mathrm{~b}$ & $61.188 \mathrm{a}$ \\
$\mathrm{CN}$ & $2.613 \mathrm{~b}$ & $8.637 \mathrm{~b}$ & $47.874 \mathrm{~b}$ \\
RR & $4.010 \mathrm{a}$ & $10.519 \mathrm{a}$ & $61.789 \mathrm{a}$ \\
\hline Dosis de potasio $\left(\mathrm{mmol} \mathrm{L}^{-1}\right)$ & & & \\
7 & $0.428 \mathrm{c}$ & $1.881 \mathrm{c}$ & $61.270 \mathrm{~b}$ \\
9 & $1.163 \mathrm{~b}$ & $5.341 \mathrm{~b}$ & $47.486 \mathrm{c}$ \\
11 & $2.809 \mathrm{~b}$ & $12.491 \mathrm{a}$ & $39.093 \mathrm{c}$ \\
13 & $6.478 \mathrm{a}$ & $15.688 \mathrm{a}$ & $79.952 \mathrm{a}$ \\
\hline Interacción & $\mathrm{n}$ & $* *$ & $* *$ \\
A*B & $\mathrm{ns}$ & \\
\hline
\end{tabular}

AR: Ácido rosmarínico; CFT: Compuestos fenólicos totales; CAET: Capacidad antioxidante equivalente Trolox; SL: Sweet Lemon; CN: Cinammon; RR: Red Rubin; A: Variedad; B: Tratamientos; ns: no significativo; **: altamente significativo. Letras distintas en columnas indican diferencias altamente significativas al $p \leq 0.01$.

\section{Contenido de compuestos fenólicos totales}

Los resultados de cuantificación de compuestos fenólicos totales (CFT) presentaron diferencias significativas $(p \leq 0.01)$ entre variedades como dosis de $\mathrm{K}$ (tabla 1$)$. Al observar los resultados por variedad se observa un porcentaje mayor en Red Rubin (18\% y 30\%) respecto de Cinammon y Sweet Lemon. De acuerdo a la concentración de K se observa que entre mayor cantidad de $\mathrm{K}$ este disponible para la planta la concentración de CFT se ve favorecida. Se encontró una interacción que indica que en alguno de los niveles de potasio tuvieron efecto diferente sobre las variedades de albahaca (fig. 1). A diferencia de los datos mostrados en la tabla 1 en la siguiente figura se muestra la relación que hay entre el tratamiento y la variedad de la planta con respecto al contenido de CFT. En la variedad Sweet Lemon la diferencia atribuible a la dosis de $\mathrm{K}$ aplicada fue significativa obteniendo el mayor valor en la dosis establecida con $13 \mathrm{mmol} \mathrm{L}^{-1}$. Para la variedad Cinammon hay un incremento en el contenido de compuestos fenólicos debido al aumento de la dosis de $\mathrm{K}$; sin embargo, en esta variedad el mejor tratamiento fue en $11 \mathrm{mmol} \mathrm{L}^{-1} \mathrm{~K}$. Finalmente, para la variedad Red Rubin se observa que hay una respuesta de aumento en la concentración de CFT en la dosis más alta.

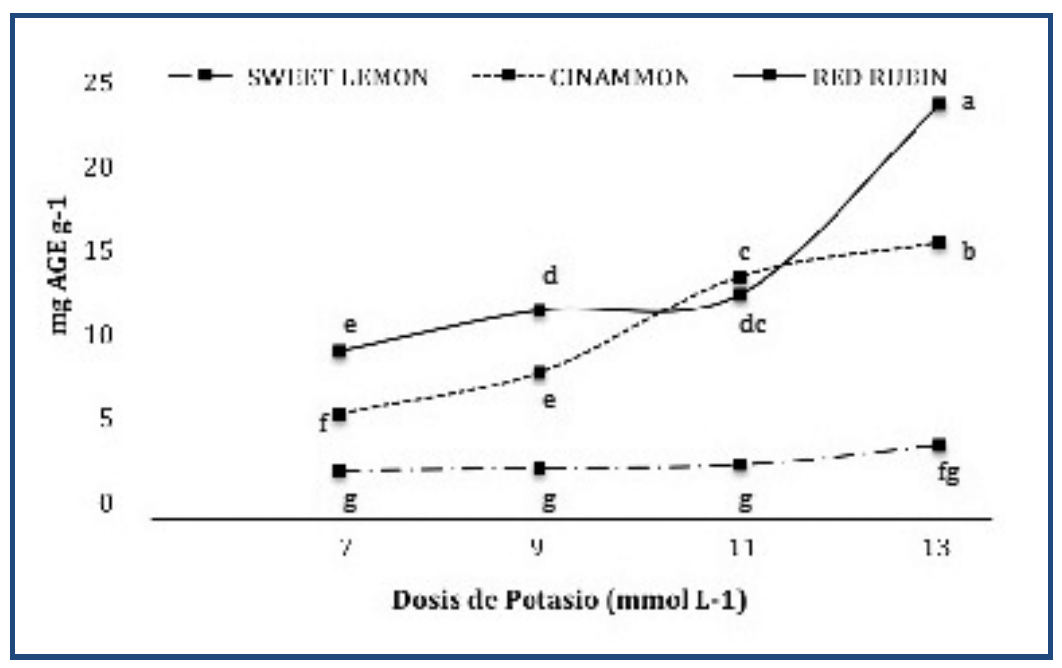

Fig. 1. Interacción de la variedad de albahaca y la dosis de potasio en el contenido de compuestos fenólicos totales. 


\section{Capacidad antioxidante total}

Los resultados obtenidos para capacidad antioxidante equivalente en Trolox (CAET) mostraron diferencias significativas entre factores (tabla 1). Para las variedades de albahaca se encontró que la variedad Red Rubin obtuvo el valor más alto y ésta fue similar a Sweet Lemon. El valor más bajo de CAET lo obtuvo la variedad Cinammon. De acuerdo a las dosis de potasio (K) el mayor resultado lo aportó la dosis de $13 \mathrm{mmol} \mathrm{L}^{-1} \mathrm{~K}$. Sin embargo, el segundo mejor tratamiento fue el de dosis control con $7 \mathrm{mmol} \mathrm{L}^{-1} \mathrm{~K}$ (tabla 1). Hubo interacción entre variedad y dosis de potasio para CAET (fig. 2), lo cual, establece que hay efecto de los tratamientos sobre las distintas variedades de albahaca utilizadas a diferencia de la tabla donde se muestra los resultados analizados por separado para los tratamientos y las tres distintas variedades. Los resultados de la interacción señalan que en la variedad SL los tratamientos 7 y 9 mmol L ${ }^{-1}$ son similares; sin embargo, para los tratamientos 11 y $13 \mathrm{mmol} \mathrm{L}^{-1}$ se incrementa la CAET de manera significativa. La variedad RR muestra un incremento de la CAET en 9 mmol $\mathrm{L}^{-1}$ pero se mantiene a $11 \mathrm{mmol} \mathrm{L}^{-1}$ e incrementa significativamente en $13 \mathrm{mmol} \mathrm{\textrm {L } ^ { - 1 }}$. La variedad $\mathrm{CN}$ muestra una respuesta similar a la variedad $\mathrm{RR}$ pero con valores inferiores a esta.

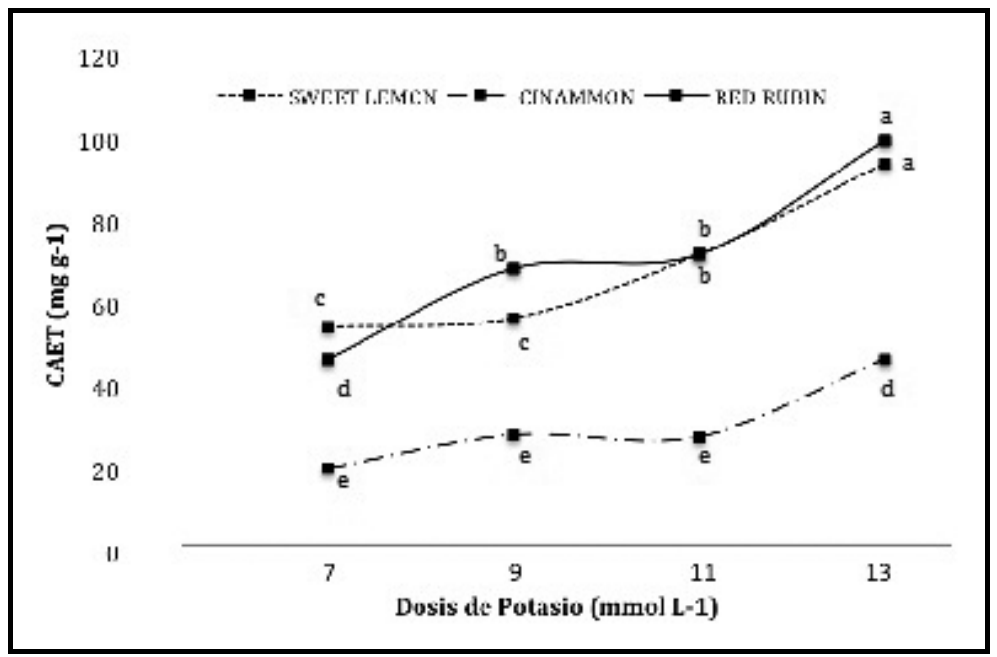

Fig. 2. Interacción de la variedad de albahaca y la dosis de potasio en la capacidad antioxidante total.

\section{DISCUSIÓN}

Los resultados para ácido rosmarínico muestran valores inferiores a los obtenidos por Muñoz et al. (2013) quienes evaluaron distintos métodos de secado y obtuvieron un promedio de $20 \mu \mathrm{g}$ $\mathrm{mg}^{-1}$ en romero, lo cual, hace referencia a que esta planta es una mejor fuente para la extracción de este compuesto. En un estudio en albahaca cuantificaron la acumulación de ácido rosmarínico en cultivo in vitro y reportaron un promedio de $15 \mu \mathrm{g} \mathrm{mg}^{-1}$ de muestra en peso seco (Kintzios, Makri, Panagiotopoulos, \& Scapeti, 2003), valores que están por encima de la media obtenida en este trabajo. En otro estudio, donde se hizo una comparación del contenido de AR en distintos estadios vegetativos de albahaca in vitro se reportaron datos con promedios de 45 $\mathrm{mg} \mathrm{g}^{-1}$ de muestra en peso seco (Kiferle et al., 2011). Otros estudios indican que de acuerdo a las condiciones de crecimiento, manejo agronómico, disponibilidad de agua, disponibilidad de nutrientes así como la variedad pueden influir en el contenido de ácido rosmarínico (Moghaddam \& Mehdizadeh, 2015). De acuerdo a Nguyen et al (2010) las concentraciones de ácido rosmarínico que determinaron oscilaron entre $2.4 \mathrm{mg} \mathrm{g}^{-1}$ y $12.8 \mathrm{mg} \mathrm{g}^{-1}$ en PS para los distintos cultivares que analizaron, demostrando que los cambios en la tasa de potasio pueden tener un impacto significativo en la composición fenólica y la capacidad antioxidante de las hojas de albahaca; ya que, la tasa más alta de potasio produjo concentraciones más altas de 
ácido rosmarínico y chicórico en los cultivares de albahaca, esto último atribuido a la vía del shikimato ya que los ácidos fenólicos son los productos finales estables de las vías bioquímicas asociadas con la asignación de recursos. El ácido rosmarínico es el compuesto fenólico de mayor presencia en albahaca. Así mismo, esto puede estar relacionado con la pigmentación oscura que se presenta en Red Rubin, debido a que sintetiza mayor cantidad de metabolitos secundarios (Kwee \& Niemeyer, 2011).

El contenido de CFT obtenido en este trabajo está dentro de los parámetros reportados por diversos autores. Por ejemplo, Flanigan y Niemeyer (2014) analizaron en 10 cultivares de albahaca el contenido de compuestos fenólicos totales y reportaron valores de 13.1 a $26.9 \mathrm{mg} \mathrm{g}^{-1}$ en peso seco. Kwee y Niemeyer (2011) encontraron en 15 cultivares de albahaca rangos de 3.47 a $17.58 \mathrm{mg} \mathrm{g}^{-1}$ en peso seco. Nguyen et al. (2010) obtuvieron valores de 7 a $16 \mathrm{mg} \mathrm{g}^{-1} \mathrm{de}$ muestra en diferentes cultivares. Anteriormente, Shan et al. (2005) encontraron valores de 36 mg AGE $\mathrm{g}^{-1}$ PS en albahaca. En dichos estudios es probable que estas diferencias se deban a las variaciones estacionales y condiciones de crecimiento como temperatura, humedad, luz y genotipo, que previamente se ha demostrado que afectan la composición química de la albahaca (Hussain et al., 2008). Añadiendo que la albahaca es conocida por su variabilidad en composición química (Simon \& Morales, 1999).

El contenido de ácido rosmarínico, así como el contenido de compuestos fenólicos totales obtenido en este trabajo fue diferente para cada una de las variedades, pudiéndose atribuir a las diferencias genotípicas de cada variedad, así mismo hubo diferencia de respuesta en la captación y uso del potasio en éstas. Posiblemente, en el desarrollo particular de cada variedad se producen más fotosintatos a medida que hay variaciones en las tasas de potasio; por lo tanto, las concentraciones de estos compuestos pueden incrementar debido al exceso de carbono fijo que se produce por la vía de los fenilpropanoides (Crozier et al., 2007). Además, el potasio es un activador de enzimas implicadas en la fotosíntesis y biosíntesis del almidón (Amtmann, Troufflard, \& Armengaud, 2008), así como de la síntesis de compuestos fenólicos debido a que activan rutas del metabolismo secundario a través de la enzima fenilalanina amonioliasa (PAL) (Koca \& Karaman, 2015). Así pues, el potasio se relaciona con el aumento del crecimiento de las plantas, así como el incremento de metabolitos secundarios.

La CAET puede tener rangos de variacion muy amplios entre variedades, lo cual se observó en este estudio. Flanigan y Niemeyer (2014), encontraron valores de $24.6 \mathrm{mmol} 100 \mathrm{~g}^{-1} \mathrm{PS}$ en albahaca Purple Ruffles y 34.8 mmol $100 \mathrm{~g}^{-1}$ PS en albahaca Purple Delight, las cuales son de color púrpura y los valores fueron superiores a los valores reportados para otras variedades de albahaca en otros trabajos, como Surveswaran et al. (2007) quienes obtuvieron en albahaca dulce $7.0 \mathrm{mmol} 100 \mathrm{~g}^{-1}$ en peso seco y el valor más alto que reportaron Kwee \& Niemeyer (2011) fue de $11.5 \mathrm{mmol} 100 \mathrm{~g}^{-1}$ para albahaca variedad Gecofure. En las variedades estudiadas se observa un incremento de la CAET de acuerdo al aumento del K; sin embargo, hay respuesta diferencial a las dosis utilizadas y a la concentración final del metabolito, evidenciándose que el $\mathrm{K}$ influye en la acumulación de compuestos antioxidantes en albahaca de acuerdo al genotipo de la planta.

\section{CONCLUSIONES}

Para incrementar el contenido de ácido rosmarínico y fenólicos totales en albahaca se utilizaron distintas dosis de potasio $(\mathrm{K})$. El contenido de ácido rosmarínico, fenólicos totales y la capacidad antioxidante incrementa de manera diferencial entre las variedades de albahaca estudiada. La variedad con mayor contenido de ácido rosmarínico, fenólicos totales y capacidad antioxidante fue Red Rubin a $13 \mathrm{mmol} \mathrm{L}^{-1}$. El K influye en la acumulación de compuestos antioxidantes en albahaca de acuerdo al genotipo de la planta (Nguyen et al., 2010), debido a que ésta presenta una química muy compleja y por ello los resultados obtenidos son diferentes entre variedad, por lo cual se sugiere realizar estudios de caracterización molecular para 
estudiar el gen que expresa el acido rosmarínico y confirmar que el $\mathrm{K}$ actúa de manera directa en un punto de la ruta metabólica de la planta y eleva la expresión del gen para aumentar el contenido del AR. Por otro lado, el potencial de producción de albahaca en México es amplio, por lo que el incremento de su producción y calidad nutracéutica podría ser una alternativa para abrir nuevos mercados enfocados a la industria alimentaria y medicinal.

\section{LITERATURA CITADA}

Ainsworth, E. A., \& Gillespie, K. M. (2007). Estimation of total phenolic content and other oxidation substrates in plant tissues using Folin-Ciocalteu reagent. Nature Protocols, 2(4), 875-877. https://doi.org/10.1038/nprot.2007.102

Amtmann, A., Troufflard, S., \& Armengaud, P. (2008). The effect of potassium nutrition on pest and disease resistance in plants. Physiologia Plantarum, 133(4), 682-691. https://doi.org/10.1111/j.1399-3054.2008.01075.x

Aubad, P., Rojano, B., \& Lobo T.E. (2007). Actividad antioxidante en musgos. Scientia Et Technica, 33(33), 23-26.

Crozier, A., Jaganath, I. B., \& Clifford, M. N. (2007). Phenols, Polyphenols and Tannins: An Overview. Plant Secondary Metabolites: Occurrence, Structure and Role in the Human Diet, (i), 1-24. https://doi.org/10.1002/9780470988558.ch1

Flanigan, P. M., \& Niemeyer, E. D. (2014). Effect of cultivar on phenolic levels, anthocyanin composition, and antioxidant properties in purple basil (Ocimum basilicum L.). Food Chemistry, 164, 518-526. https://doi.org/10.1016/j.foodchem.2014.05.061

Hussain, A. I., Anwar, F., Hussain Sherazi, S. T., \& Przybylski, R. (2008). Chemical composition, antioxidant and antimicrobial activities of basil (Ocimum basilicum) essential oils depends on seasonal variations. Food Chemistry, 108(3), 986-995. https://doi.org/10.1016/ j.foodchem.2007.12.010

José, J., Pérez, R., Amador, B. M., Garibay, A. N., Diéguez, E. T., María, I., ... Cuervo, L. (2014). (Ocimum basilicum L.) EN CONDICIONES DE SALINIDAD Growth and Development of Basil (Ocimum basilicum L.) Varieties Under Conditions of Salinity, $35-45$.

Kiferle, C., Lucchesini, M., Mensuali-Sodi, A., Maggini, R., Raffaelli, A., \& Pardossi, A. (2011). Rosmarinic acid content in basil plants grown in vitro and in hydroponics. Open Life Sciences, 6(6). https://doi.org/10.2478/s11535-011-0057-1

Kintzios, S., Makri, O., Panagiotopoulos, E., \& Scapeti, M. (2003). In vitro rosmarinic acid accumulation in sweet basil (Ocimum basilicum L.), 1, 405-408.

Koca, N., \& Karaman, Ş. (2015). The effects of plant growth regulators and 1-phenylalanine on phenolic compounds of sweet basil. Food Chemistry, 166(June), 515-521. https://doi.org/10.1016/j.foodchem.2014.06.065

Kwee, E. M., \& Niemeyer, E. D. (2011). Variations in phenolic composition and antioxidant properties among 15 basil (Ocimum basilicum L.) cultivars. Food Chemistry, 128(4), 1044-1050. https://doi.org/10.1016/j.foodchem.2011.04.011

Lee, J., \& Scagel, C. F. (2009). Chicoric acid found in basil (Ocimum basilicum L.) leaves. Food Chemistry, 115(2), 650-656. https://doi.org/10.1016/j.foodchem.2008.12.075

López-Blancas, E., Martínez-Damián, M. T., Colinas-León, M. T., Bautista-Boñuelos, C., Martínez-Solís, J., \& Rodríguez-Pérez, J. E. (2014). Actividad antioxidante y enzimática de albahaca "Nufar" (Ocimum basilicum L.) almacenada en refrigeración. Agronomía Mesoamericana, 25(2), 255. https://doi.org/10.15517/am.v25i2.15428

Moghaddam, M., \& Mehdizadeh, L. (2015). Variability of total phenolic, flavonoid and rosmarinic acid content among Iranian basil accessions. LWT - Food Science and Technology, 63(1), 535-540. https://doi.org/10.1016/j.lwt.2015.03.068

Muñoz-Muñoz, J. L., Garcia-Molina, F., Ros, E., Tudela, J., García-Canovas, F., \& RodriguezLopez, J. N. (2013). Prooxidant and antioxidant activities of rosmarinic acid. Journal of Food Biochemistry, 37(4), 396-408. https://doi.org/10.1111/j.17454514.2011.00639.x 
Recibido:

30/octubre/2017

Aceptado:

13/agosto/2018
Nguyen, P. M. and E. D. N. (2008). Effects of nitrogen fertilization on phenolic composition and antioxdant properties of basil (Ocimum basilicum L.). Brown Working Papers in the Arts of Sciences, 8, 1-25. https://doi.org/10.1021/jf801485u

Nguyen, P. M., Kwee, E. M., \& Niemeyer, E. D. (2010). Potassium rate alters the antioxidant capacity and phenolic concentration of basil (Ocimum basilicum L.) leaves. Food Chemistry, 123(4), 1235-1241. https://doi.org/10.1016/j.foodchem.2010.05.092

Nurzynska-Wierdak, R. (2013). Essential oil composition of sweet basil cultivars as affected by nitrogen and potassium fertilization. Turkish Journal of Agriculture and Forestry, 427-436. https://doi.org/10.3906/tar-1203-43

Parr, A. J., \& Bolwell, G. P. (2000). Phenols in the plant and in man. The potential for possible nutritional enhancement of the diet by modifying the phenols content or profile. Journal of the Science of Food and Agriculture, 80(7), 985-1012. https://doi.org/10.1002/(SICI)1097-0010(20000515)80:7<985::AID-JSFA572>3.0.CO;2-7

Ramos De Leon, N., Sanabria Chópite, M. E., Rodríguez González, D. A., \& Ulacio, D. (2012). Efecto del extracto etanólico de albahaca genovesa (Ocimum basilicum var. genovese) sobre Cercospora apii Fressen y el tizón temprano del celery (Apium graveolens). Revista Cientifica UDO Agrícola, 12(2), 472-478.

Sanchez, C., \& Lucero, J. M. (2012). Nicho de mercado de especies aromáticas orgánicas tipo gourmet. Baja California Sur.

SAS (Statistical Analisys System) Institute. (2004). Cary NC. USA.

Shan, B., Cai, Y. Z., Sun, M., \& Corke, H. (2005). Antioxidant Capacity of 26 Spice Extracts and Characterization of Their Phenolic Constituents Antioxidant Capacity of 26 Spice Extracts and Characterization of Their Phenolic Constituents. Journal of Agricultural and Food Chemistry., (53), 7749-7759. https://doi.org/10.1021/jf051513y

SIAP. (2015). Servicio de Información Agroalimentaria y Pesquera. Retrieved from http:/www.sagarpa.gob.mx/quienesomos/datosabiertos/siap/Paginas/default.aspx

Simon, J., \& Morales, M. (1999). Basil: a source of aroma compounds and a popular culinary and ornamental herb. Perspectives on New Crops and New Uses., (16), 499-505. Retrieved from https://www.hort.purdue.edu/newcrop/proceedings 1999/pdf/v4-499.pdf

Singh, K., Singh, P. P., Beg, S. U., Kumar, D., \& Patra, D. D. (2004). Effect of NPK fertilizers on growth, oil yield and quality of French basil (Ocimum basilicum L.). Journal of Spices and Aromatic Crops, 13(1), 52-54.

Steiner, A. A. (1961). A Universal Method for preparing nutrient solutions of a certain, (2), 134-135.

Surveswaran, S., Cai, Y. Z., Corke, H., \& Sun, M. (2007). Systematic evaluation of natural phenolic antioxidants from 133 Indian medicinal plants. Food Chemistry, 102(3), 938 953. https://doi.org/10.1016/j.foodchem.2006.06.033

Treutter, D. (2006). Significance of flavonoids in plant resistance: A review. Environmental Chemistry Letters, 4(3), 147-157. https://doi.org/10.1007/s10311-006-0068-8 